\title{
VALSALVA MANOUEVRE;
}

Efficacy in the treatment of Eustachian catarrh.

\section{Dr Muhammad Khalid Azam Khan, Mr. Shahid Bin Faiz, Mr. Atif Rafique}

ABSTRACT... Objective: To assess the efficacy of Valsalva maneuver in the treatment of Eustachian catarrh. Study design: Quasi Experimental Study. Place and duration of study: Frontier corp Hospital (Quetta) and Sheikh Khalifa Bin Zayad Al Nahyan Hospital (Muzzafarabad) from December 2010 to May 2011. Patients and methods: Total of sixty patients of Eustachian catarrh were randomly assigned to two treatment groups. Group A was given Antibiotics, Antihistamine, systemic and nasal decongestants and group B was advised Valsalva maneuver in addition to the above mention treatment. Relief in aural fullness (assessed through tympanometry) and hearing improvement (assessed through audiometry) were compared between these two groups at the end of three weeks of treatment. Results: Audiogram done after three weeks of treatment showed statistically significant hearing and middle ear pressure improvement (assessed through audiogram and tympanogram respectively) in group B.

Key words: Valsalva maneuver, Eustachian tube, Audiogram, Tympanogram.

\section{Article Citation}

$\checkmark \quad$ Khan MKZ, Faiz SB, Rafique A. Efficacy of valsalva manouevre in the treatment of Eustachian catarrh. Professional Med J Feb 2013;20(1): 121-125.

\section{INTRODUCTION}

The Eustachian tube is a narrow tube that connects the middle ear with nasopharynx. In adults it is about 3-4 $\mathrm{cm}$ long. The Eustachian tube is anatomically different in children, where it is more horizontal, shorter and wider making them prone to ascending infections. By the age of 7 years, the tube changes to adult type. Eustachian tube dysfunction is failure of the Eustachian tube to open sufficiently during swallowing or yawning, causing a difference in air pressure inside and outside the middle ear. Allergy, upper respiratory tract infections, adenoids, rapid altitude changes, narrow Eustachian tube, and tumors in the nasopharynx also cause Eustachian tube dysfunction. Much of Eustachian tube pathology relates to mucosal disease and associated hypertrophy.

Patient has feeling of aural fullness and deafness. The symptoms of tubal catarrh may persist for a week and even longer. Many authors have concluded that Eustachian tube dysfunction is the most important precipitating pathology in the development of Otitis media $^{1-5}$. However the treatment options are limited. Tympanotomy and insertion of the ventilation tube is done when the medical treatment fails to restore the patency of the Eustachian tube but the symptoms recur after the removal of ventilation tube $e^{6-7}$.

Examination of the patient usually reveals inflamed mucosa of the upper respiratory track. The tympanic membrane is usually congested with blood vessels radiating from the handle of Malleus like spokes of a wheel (cart wheel appearance), with distorted cone of light and decrease mobility. Endoscopic evaluation and pneumatic otoscopy is very helpful. Eustachian tube functions can be tested qualitatively by Valsalva maneuver, politerization or Eustachian tube catheterization and quantitatively by tympanometry audiometery and Tympanometry. The Valsalva maneuver is performed by moderately forceful attempted exhalation against a closed airway by closing the mouth and nose. The technique is named after Antonio Maria Valsalva, the 17th Century physician and anatomist from Bologna, whose principal scientific interest was the human ear. He described the Eustachian tube and the maneuver to test its patency. 
Yagi and colleagues evaluated the patency of the Eustachian tube using a fiberoptic endoscope and a photoelectric device (phototubometry) ${ }^{8}$. javascript:showcontent('active','references'); Using videoendoscopy of the ear, Poe and colleagues assessed tubal function in adults and observed various disease processes such as inflammation of the tube and patulous dysfunction.Upper respiratory track infection and associated Eustachian tube dysfunction is quite common in our country causing multiple social and behavioral problems to the school going children. This study is aimed at determining the efficacy of Valsalva maneuver in patients of Eustachian catarrh.

\section{PATIENTS AND METHOD}

Approval was taken from hospital ethical committee.The study was conducted in Frontier corp Hospital (Quetta) and Sheikh Khalifa Bin Zayad Al Nahyan Hospital (Muzzafarabad) from December 2010 to may 2011.

It comprised of 60 patients of Eustachian catarrh reporting in ENT OPD. Patients above the age of 12 years were included in the study so that anatomical difference of Eustachian tube in children is not a factor. All patients were having associated upper respiratory tract infection .Patients with associated pathology like gross DNS, nasal mass including nasal polyps, perforated ear drum, Tympanosclerosis, previous conductive or sensorineural deafness and chronic adhesive otitis media were excluded from the study. A detailed history was taken from all the patients followed by complete ENT examination including pneumatic Otoscopy and nasal Endoscopy. Informed consent was obtained from all patients after explaining advantages of the Valsalva maneuver. Tympanogram and audiogram were obtained on their first visit to opd. All patients (in both groups) were given tablet panadol CF (Paracetamol, chlorphenaramine and pseudoephederine) and tablet Augmentin (Coamoxiclave) for 7 days along with nasal decongestants Xynoscine
(Oxymetazolin) for three weeks. Group B was advised to perform Valsalva maneuver thrice daily for three weeks in addition to the above mentioned treatment. Valsalva maneuver is practically demonstrated to the patients so that they can perform the maneuver effectively.

Patients were reviewed after three weeks of treatment. Complete ENT examination was performed along with Tympanogram and Audiogram. Hearing improvement of more than $15 \mathrm{db}$ is considered significant and change of type $\mathrm{C}$ curve (indicating negative middle ear pressure) to type A curve (normal middle ear pressures) was considered significant.

Data was analyzed using SPSS 14. The difference between the two was compared using paired sample ttest as the test of significance. $P$ value less than 0.05 was considered significant.

\section{RESULTS}

Total of 60 patients of Eustachian catarrh reporting in hospital were studied over a period of six months. The patients of Eustachian catarrh were randomly divided into two treatment groups. All patients (in both groups) were treated for infection and inflammation with Antibiotics, Antihistamine and Decongestants. There were $24(80 \%)$ male and 6(20\%) female patients in group A whereas there were $26(86 \%)$ male and $4(13 \%)$ female patients in group $B$. In group $A$ the mean age of the patient was 30 years ranging from 14 to 52 years and the mean age in group B was 33 years ranging from 16 to 48 years. Pre treatment Audiograms and Tympanograms were kept as a record. Audiogram and Tympanograms done after three weeks of treatment showed that there was statistically significant hearing and middle ear pressure improvement in group $B$ ( $P$ value less than 0.05$)$. In group B, 24 (80\%) patients showed improvement of more than $15 \mathrm{~dB}$ in their Audiograms as compared to 5 (16.6\%) patients in group A which showed that our results are significant (Table I) . Similarly 26 (86.66\%) 
patients had improved Tympanogram in group $B$ as compared to $5(16.66 \%)$ patients in group $A$ again proving the significance of the study (Table II).

\begin{tabular}{|l|c|c|}
\hline & $\begin{array}{c}>15 \mathrm{~dB} \\
\text { improvement in } \\
\text { audiogram }\end{array}$ & $\begin{array}{c}<15 \mathrm{~dB} \\
\text { improvement in } \\
\text { audiogram }\end{array}$ \\
\hline $\begin{array}{l}\text { Group } A \\
(\mathrm{n}=30)\end{array}$ & $5(16.66 \%)$ & $25(83.33 \%)$ \\
\hline $\begin{array}{l}\text { Group B } \\
(\mathrm{n}=30)\end{array}$ & $24(80 \%)$ & $6(20 \%)$ \\
\hline & $P<0.05$ & \\
\hline
\end{tabular}

Table-I. Percentage of patients having significant improvement in audiogram ( $>15 \mathrm{~dB}$ )

\begin{tabular}{|l|c|c|}
\hline & $\begin{array}{c}\text { Improved } \\
\text { Tympanogram } \\
\text { (type C to type A) }\end{array}$ & $\begin{array}{c}\text { No improvement } \\
\text { in Tympanogram } \\
\text { (Type C) }\end{array}$ \\
\hline $\begin{array}{l}\text { Group A } \\
(\mathrm{n}=30)\end{array}$ & $5(16.66 \%)$ & $25(83.33 \%)$ \\
\hline $\begin{array}{l}\text { Group B } \\
(\mathrm{n}=30)\end{array}$ & $26(86.66 \%)$ & $4(13.33 \%)$ \\
\hline & $P<0.05$ & \\
\hline
\end{tabular}

Table-ll. Percentage of patients having significant improvement in tympanogram (from type C to A)

\section{PATIENT OUTCOME}

All patients responded to medical treatment as far as infection and inflammation was concerned.

\section{DISCUSSION}

Eustachian catarrh is a frequently presented disease in ENT opd. Patients usually present with deafness and aural fullness. Complete ENT examination including pneumatic otoscopy and Endoscopy is required to reach the diagnosis. Main stay of the treatment is medical and restoring the patency of Eustachian tube. Medical treatment is cost effective ${ }^{9}$ and surgery in the form of Myringotomy and insertion of the ventilation tube is reserved for those cases which fail to resolve with medical treatment. Screening tympanometry was carried out in 243 school children and it was found that

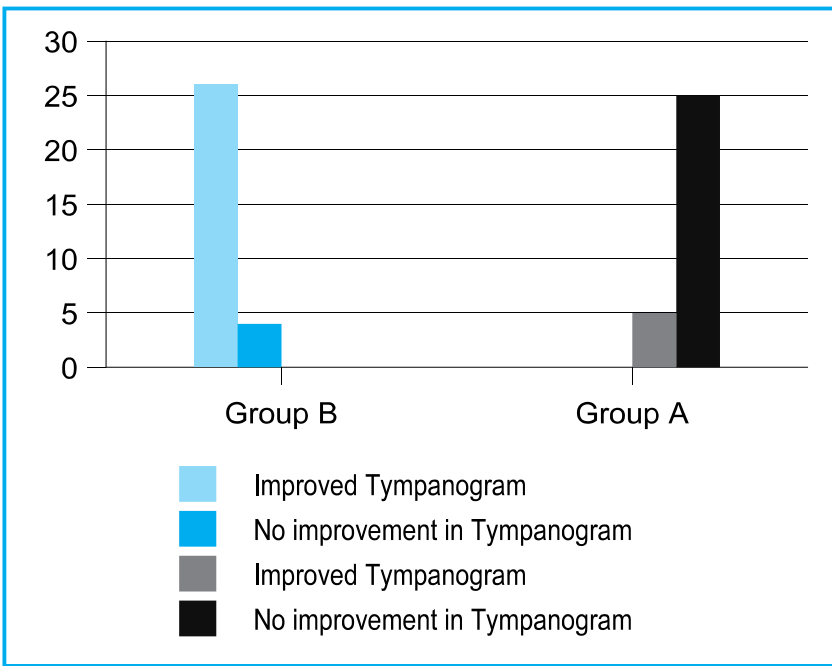

\section{Graphical presentation of improvement in audiogram in group A \& B}

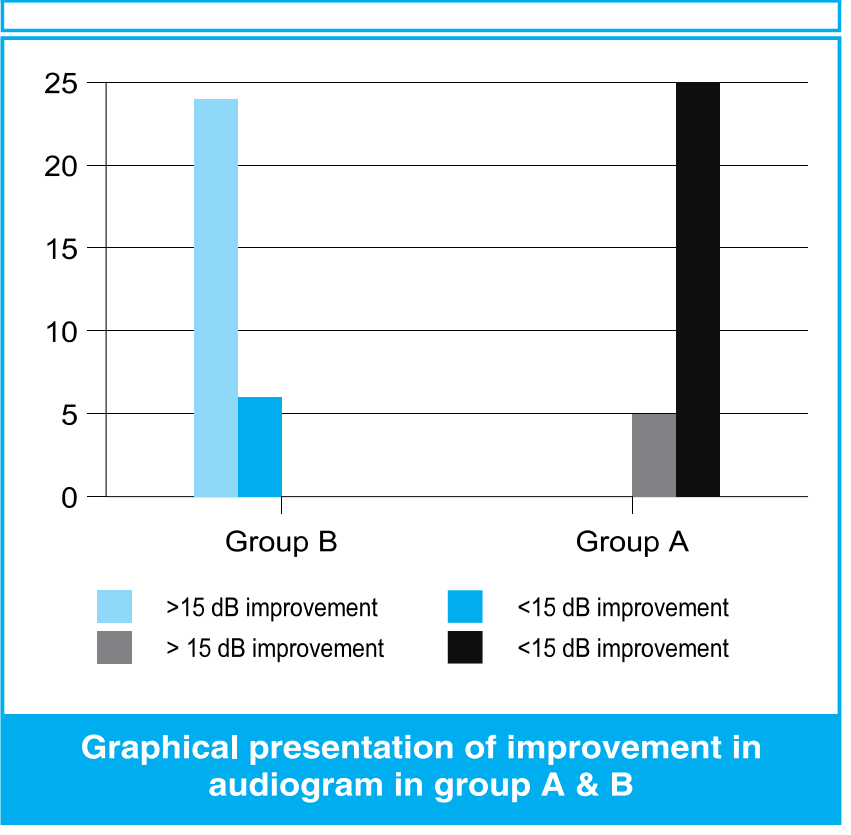

$36 \%$ of children were found to have Eustachian tube dysfunction ${ }^{10}$.The Eustachian tube dysfunction and otitis media with effusion are quite prevalent in our country and due to limited access of the patients to doctors, this entity is probably under diagnosed. This study demonstrates the effectiveness of Valsalva maneuver in the treatment of Eustachian catarrh.

Majority of the patients were male as the study was carried out in a paramilitary set up. All patients were 
given same treatment to exclude any confounding factor affecting the results of the study. Patients were evaluated through audiogram and tympanogram for improvement in hearing and middle ear pressure respectively.

Our results show that in patients with Eustachian catarrh, Valsalva maneuver plays a vital role in relieving the symptoms of aural fullness and deafness assessed through Audiogram and Tympanogram. These results are supported by Caffier and collegues ${ }^{11}$ who studied the Impact of Laser Eustachian Tuboplasty on middle ear ventilation, hearing, and tinnitus in patients with Eustachian tube dysfunction. They perform trans nasal laser Eustachian Tuboplasty and found a significant improvement of Eustachian tube functions. They concluded that those patients who performed Valsalva maneuver had more satisfaction and symptomatic improvement than patients without successful Valsalva maneuver. In Another small prospective study by Dr. Poe, Dr. Metson, and Dr. Kujawski in which they carried out laser Eustachian Tuboplasty, they found Laser safe and efficacious in the treatment of intractable Eustachian tube dysfunction.

In a prospective study by Herbert Silverstein ${ }^{12}$, the role of topical steroid was studied in 11 patients with Eustachian tube dysfunction. All patients had previously been treated with medical therapy and surgical middle ear ventilation without success. A micro wick was placed directly in the Eustachian tube orifice in nasopharynx. Patients received 3 drops of Dexamethasone three times a day for 04 weeks. Patients had improvement in aural pressure and fullness confirmed through tympanogram. Aural fullness or pressure resolved completely in eight patients (72.7\%). and audiogram demonstrated a 55\% reduction in the mean air-bone gap.

Efficacy of politerization on Eustachian tube catarrh was studied on fourteen patients by Shlomo ${ }^{13}$. Patients were managed with politerization twice a week for six weeks and after that they were evaluated through tympanometry and it was found that they had significant improvement in their mean Tympanometric peak pressure which was not observed in control subjects.

Lermoyz has used hot air in the treatment of Eustachian catarrh where he used hot air at temperature $160-195^{\circ} \mathrm{F}$. in order to reduce the congestion and secretions of the mucus membrane. After anaesthetizing the mucosa, hot air was inflated through a specialized apparatus to the opening of Eustachian tube in nasopharynx. The treatment session last for 1-2 minutes and done 5-6 times in 1-2 days period. The purpose of the treatment was to relieve the congestion and restore the patency of the Eustachian tube.

Curtis H. Muncie ${ }^{14}$ developed a manipulative technique to relieve Eustachian tube dysfunction. He inserted a gloved right index finger into the patient's mouth, placing the finger against the inferior part of the posterior pillar of the palatine tonsil. Moving the finger tip up and slightly lateral to the Rosenmüller fossa, posterior to the opening of the Eustachian tube, the physician should apply a pumping motion with the finger pad to lyse any adhesions and, ultimately, restore the Eustachian tube opening. However, this technique may cause gagging and can be traumatic for children.

The results of the study show that Valsalva maneuver has a significant role in the management of Eustachian catarrh and it should be performed effectively in adult patients where it is not settling. Nasopharyngoscopy should be performed to exclude malignancy in nasopharynx. In our set up children are often suffering from Eustachian tube dysfunction, having social and behavioral problems and this area needs further work up. Further studies at larger level need to be planned especially in children to validate these findings. 


\section{CONCLUSIONS}

Eustachian tube dysfunction is a commonly presented disease in ENT and is the cause of social and behavioral problem in school going children and adults. proper diagnosis and management is required Valsalva maneuver is old time tested way of relieving the Eustachian tube obstruction and effective demonstration is required to achieve good results. Just by adding this simple maneuver to the standard treatment can efficiently cure the individual thus reducing the morbidity. Further research work at a larger level is required in this field especially in children to address the problem in an effective and simple way.

\section{Copyright@ 31 0ct, 2012.}

\section{REFERENCES}

1. Sade J, Ar A. Middle ear and auditory tube: Middle ear clearance, gas exchange, and pressure regulation. Otolaryngol Head Neck Surg 1997; 116:499-524.

2. Bluestone CD, Klein J0. Otitis Media in Infants and Children. 2nd ed. Vol. 1. Philadelphia: W.B. Saunders, 1995:5-38.

3. Tos $M$. The intraluminal obstructive pathogenic concept of eustachian tube in secretory otitis media. In: Sade J, ed. Basic Aspects of the Eustachian Tube and Middle Ear Diseases. Amsterdam: Kugler and Ghedini, 1991:327-33.

4. Bluestone CD, Paradise JL, Beery QC. Physiology of the eustachian tube in the pathogenesis and management of middle ear effusions. Laryngoscope 1972; 82:1654-70.

5. Cantekin El, Bluestone CD, Parkin LP. Eustachian tube ventilatory function in children. Ann Otol Rhinol Laryngol 1976; 85(2 Suppl 25 Pt2):171-7.
6. Schuknecht HF, Zaytoun GM, Moon CN, Jr. Adult onset fluid in the tympanomastoid compartment. Diagnosis and management. Arch Otolaryngol 1982; 108:75965.

7. Lamp CB, Jr. Chronic secretory otitis media: Etiologic factors and pathologic mechanisms. Laryngoscope 1973; 83:276-91

8. Yagi N, Haji T, and Honjo I. Eustachian tube patency detected by a photoelectric method. The Laryngoscope 1987; 97: 732-736

9. Rashid D, Ahmed B, Malik SM, Rahat ZM, Malik KZ. Otitis Media with effusion-cost effective options. $J$ Coll Physicians Surg Pak. May 2002; 12(5):274-6.

10. Ali L, Baloch GR, Khan MA, Zahra S. Screening Tympanometry in school children: criteria for detection of middle ear effusion. Pak Paed J. Mar 2004; 28(1):21-4.

11. Caffier, Philipp P, Sedlmaier, Benedikt, Haupt, Heidemarie, Göktas, Önder, Scherer, Hans, Mazurek, Birgit. Impact of Laser Eustachian Tuboplasty on Middle Ear Ventilation, Hearing, and Tinnitus in Chronic Tube Dysfunction. Ear and Hearing; 2011; 32;132-139.

12. Herbert S, Joshua P, Lance JE, Seth RI, Jack TH. Direct application of Dexamethasone for the treatment of chronic Eustachian tube dysfunction. Ear, nose, \& throat journal 2003; 82(1):28-32.

13. Silman S, Arick D. Efficacy of a modified politzer apparatus in management of Eustachian tube dysfunction in adults. J. Am. Acad. Audiol1999 0ct; 10(9):496-501.

14. Curtis $\mathrm{H}$, Muncie. Osteopathic manipulation for Eustachian tube dysfunction. JAOA VOL 108; 2008;260-263.
AUTHOR(S):
1. DR MUHAMMAD KHALID AZAM KHAN
Classified ENT Specialist
Sheikh Khalifa Bin Zyed Al Nahyan Hospital
Muzaffarabad.
2. MR. SHAHID BIN FAIZ
3. MR. ATIF RAFIQUE

CORRESPONDENCE ADDRESS:

Dr. Muhammad Khalid Azam Khan

Classified ENT Specialist

Sheikh Khalifa Bin Zyed Al Nahyan Hospital

Muzaffarabad.

Article received on: 28/06/2012 Accepted for Publication: $30 / 10 / 2012$ Received after proof reading: 10/12/2012 